\title{
Smart Particles Containing Multiple Rugate-structured Photonic Crystal
}

\author{
Seunghyun Jang ${ }^{\dagger}$
}

\begin{abstract}
The rugate porous silicons containing multiple photonic band gaps have been generated by applying a composite waveform summed three computer-generated pseudo-sinusoidal current waveforms and exhibit three sharp photonic band gaps in the optical reflectivity spectrum. Generated multiple rugate porous silicons display three rugate peaks corresponding to the each of the sine components varied from $0.42,0.36$, and $0.30 \mathrm{~Hz}$, with a spacing of $0.06 \mathrm{~Hz}$ between each sine component. The resulting rugate PSi films have been removed from the silicon substrate by applying an liftoff current and are then made into particles by ultrasono-method in a organic solution. The sensing experiments using these particles for organic solvents such as toluene, hexane, acetone, and methanol have been achieved. Condensing of organic vapors in the pores increases the refractive indices of entire particle which results a red shift in the photonic peaks.
\end{abstract}

Key words : Rugate, Sensing, Organics, Smart Particles, Porous Silicon

\section{Introduction}

The development of new technology for silicon nanomaterials to build such a device is of great interest, because it is too complex to fabricate by using conventional lithographic method. The research on the silicon nanomaterials for various applications in the fields of opto-electronics, photonics, photovoltaics ${ }^{[1]}$, field-effect transistors ${ }^{[2]}$, optical band pass filters, chemical and biological sensors or drug delivery were intensively investigated $^{[3-12]}$. Multistructured porous silicon (PSi) such as Rugate PSi has been recently investigated for use of its possible chemical and biological applications. Because of the tunable optical properties, PSi has become a widely studied and applied material in recent years. Porous silicon is also an attractive material due to its high surface area, convenient surface chemistry, and optical signal transduction capability ${ }^{[13-17]}$.

\section{Experimental Section}

\subsection{Sample Preparation}

Rugate PSi encoded three photonic band gaps were

Department of Chemistry, University of Wisconsin, Madison, 1101 University Ave., Madison, WI 53706 (USA)

${ }^{\dagger}$ Corresponding author : shjang@chem.wisc.edu

(Received : November 21, 2012, Revised : December 15, 2012, Accepted : December 21, 2012) prepared by electrochemically etching of heavily doped p++-type silicon wafer (boron doped, polished on the (100) face, resistivity; $0.8 \sim 1.2 \mathrm{~m} \Omega \cdot \mathrm{cm}$, obtained from Siltronix, Inc.). The etching solution consisted of a $3: 1$ volume mixture of aqueous $48 \%$ hydrofluoric acid (ACS reagent, Aldrich Chemicals) and absolute ethanol (ACS reagent, Aldrich Chemicals). Galvanostatic etching has been carried out in a Teflon cell using a twoelectrode configuration. A composite waveform summed three computer-generated pseudo-sinusoidal current waveforms varying between 51.5 and $74.6 \mathrm{~mA} / \mathrm{cm}^{2}(100 \mathrm{~s}$ periodicity, 30 repeats) is applied. Each sine component varies from $0.42,0.36$, and $0.30 \mathrm{~Hz}$, with a spacing of $0.06 \mathrm{~Hz}$ between each sine component. The resulting rugate PSi film has been removed from the silicon substrate by applying an lift-off current at $460 \mathrm{~mA} / \mathrm{cm}^{2}$ for $1.5 \mathrm{~min}$ and $29 \mathrm{~mA} / \mathrm{cm}^{2}$ for $1.5 \mathrm{~min}$. All samples were then washing with ethanol and dried under Ar gas. The freestanding films were then made into particles by ultrasono-method in a ethanol solution.

\subsection{Instrumentation and Data Acquisition}

Samples were illuminated with a tungsten lamp, and the reflected light spectrum was measured using an Ocean Optics S2000 CCD spectrometer fitted with a fiber optic input. The reflected light collection end of the fiber optic is positioned at the focal plane of the 
optical microscope. The morphologies of the rugate smart particles were observed with a cold field emission scanning electron microscope (FE-SEM, S-4800, Hitachi).

\section{Results and Discussion}

Photonic crystals containing multiple rugate structure result are prepared by applying a computer-generated pseudo-sinusoidal current waveform. The rugate PSi generated in this method exhibits many sharp lines in the optical reflectivity spectrum, which are able to be tuned to appear anywhere in the visible spectral range, depending on the programmed etch waveform. Rugate filters possess a sinusoidally varying porosity gradient in the direction perpendicular to the plane of the filter. The waveform used in the present work involves an individual sine component, which is represented by Equation 1.

$$
Y_{\mathrm{i}}=A_{\mathrm{i}} \sin \left(\mathrm{k}_{\mathrm{i}} \mathrm{t}\right)+\mathrm{B}
$$

Where $Y_{\mathrm{i}}$ represents a temporal sine wave of amplitude, $A_{\mathrm{i}}$; frequency, $\mathrm{k}_{\mathrm{i}}$; time, $\mathrm{t}$; applied current density, B.

Triply encoded multiple rugate structures can be etched on a silicon wafer, showing three sharp spectral lines in the optical reflectivity spectrum. all of the individual sine components are summed together to create A composite waveform summed all of the individual sine components has been created and shown in Equation 2 .

$$
Y_{\text {comp }}=A_{1} \sin \left(\mathrm{k}_{1} \mathrm{t}\right)+A_{2} \sin \left(\mathrm{k}_{2} \mathrm{t}\right)+A_{3} \sin \left(\mathrm{k}_{3} \mathrm{t}\right)+\mathrm{B}
$$

Equation 2 can be converted to an analog currenttime waveform to etch using a computer-controlled digital galvanostat.

A waveform containing three separate frequency components has been applied. The values of ki for each of the sine components are varied from 0.30 to $0.42 \mathrm{~Hz}$, with a spacing of $0.06 \mathrm{~Hz}$ between each sine component.

The values of $A_{\mathrm{i}}$ and $\mathrm{B}$ for every sine components are 11.55 and $63.05 \mathrm{~mA}$, respectively. Reflectivity spectrum of triple rugate structured PSi displayed three peaks shown in Fig. 1. Each of the main peaks in an optical reflectivity spectrum corresponds to one of the sine

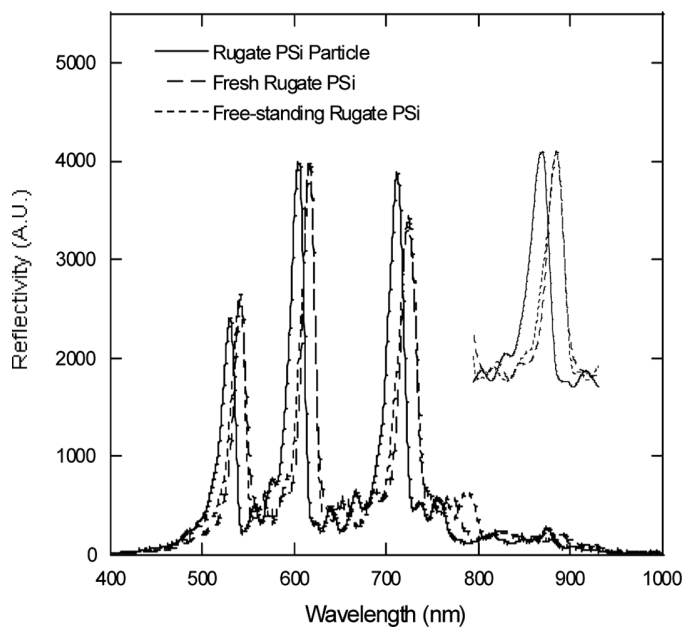

Fig. 1. Reflectivity spectra for triply encoded rugate PSi (fresh PSi, free-standing PSi, and PSi particles)

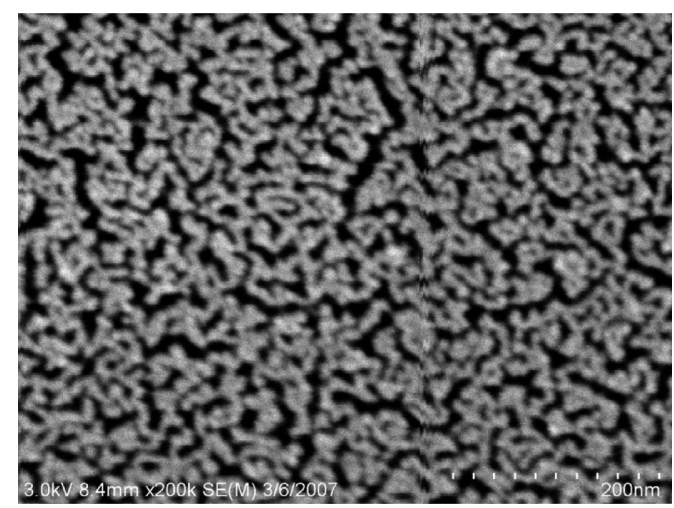

Fig. 2. Surface FESEM image of smart particle

components of the composite waveform, indicating that the reflectivity spectrum represents the Fourier transform of the composite current-time waveform. The reflectivity of free-standing rugate PSi film occurs at the identical location. The ultrasonication of PSi films results the reflectivities shifted to shorter wavelengths by $10.37,11.19,11.44 \mathrm{~nm}$, respectively.

The surface images of the smart particles indicate that the distribution of the pore size is even and that good porosity is retained with no destruction of the porous structure during the ultrasonication. The particle size varied depending on the sonication time. Fig. 3 indicated that the prepared smart particles had cylindrical macropores. The pore shape, pore size, and orentation of porous silicon layers depend on the surface orentation and doping level and type, temperature, the current 


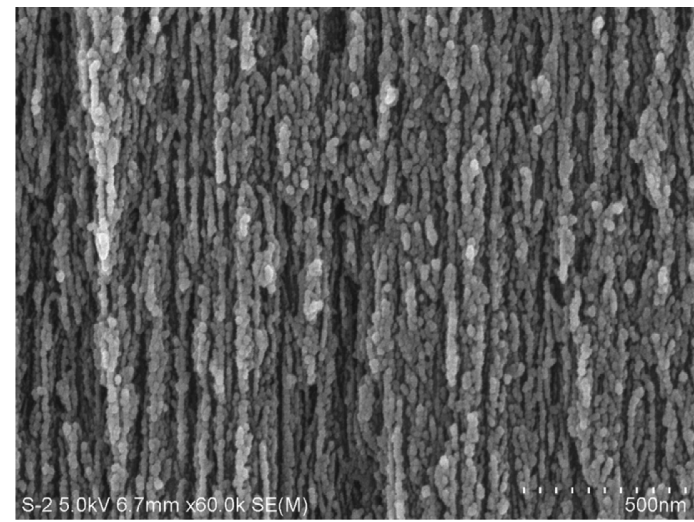

Fig. 3. Cross-sectional SEM image of smart particle

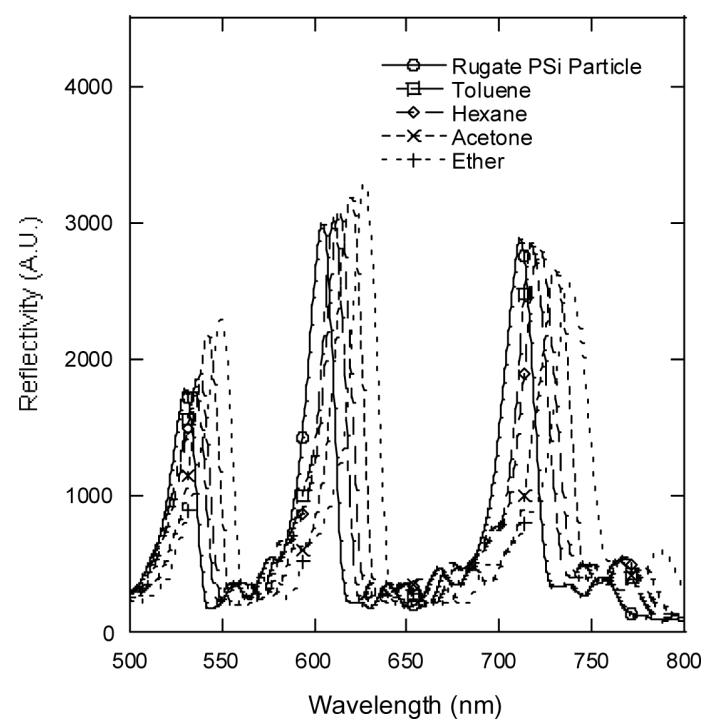

Fig. 4. Optical reflectivity spectrum of smart particles in air (solid line) and air containing organic vapors (dotted line).

density, and the composition of the etching solution.

Triply encoded smart particles have been subjected to expose to the vapor of organics such as toluene, hexane, acetone, and diethyl ether. When the smart particles are exposed to the organic vapors, capillary condensation causes the reflectivity spectrum of the smart particles to shift to longer wavelengths shown in Fig. 4.

Such a large red shift is characteristic of an increase in the average refractive index of the multilayers, consistent with the replacement of a significant amount of empty pore volume with analyte.

Table 1 and 2 show the reflection maxima and red-
Table 1. Reflection maxima for the triple rugate structured PSi under the exposure of organic vapors

\begin{tabular}{cccc}
\hline & peak 1 $(\mathrm{nm})$ & peak 2 $(\mathrm{nm})$ & peak 3 $(\mathrm{nm})$ \\
\hline particle & 531.47 & 605.52 & 712.15 \\
toluene & 536.48 & 611.12 & 718.21 \\
hexane & 538.27 & 614.27 & 723.26 \\
acetone & 543.27 & 620.55 & 731.64 \\
ether & 550.40 & 627.86 & 739.32 \\
\hline
\end{tabular}

Table 2. Red-shift of reflection maxima for the triple rugate structured PSi under the exposure of organic vapors

\begin{tabular}{cccc}
\hline & peak 1 $(\mathrm{nm})$ & peak 2 $(\mathrm{nm})$ & peak 3 $(\mathrm{nm})$ \\
\hline toluene & 5.01 & 5.60 & 6.06 \\
hexane & 6.80 & 8.75 & 11.11 \\
acetone & 11.80 & 15.03 & 19.49 \\
ether & 18.93 & 22.34 & 27.17 \\
\hline
\end{tabular}

shift of reflection maxima for the triple rugate structured PSi under the exposure of organic vapors. The amplitude of peak shift would depend on refractive index and vapor pressure of the analyte, and the surface characteristics and the pore diameter of PSi. Since the parameter for PSi was fixed, peak shift depended on refractive index, vapor pressure of an individual analyte, and molecular polarity as well as the surface characteristics including hydrophilicity/hydrophobicity. Even though smart particles terminated with silicon hydride functionalities were hydrophobic surface and had a much greater affinity for a hydrophobic versus a hydrophilic analyte, the smart particles displayed the most red-shifted photonic feature for the analyte having the highest vapor pressure. This result indicated that the specificity of adsorption or capillary condensation at the PSi surfaces depends dramatically on the vapor pressure.

\section{Conclusion}

Triply encoded smart particles have been exposed to the vapor of organics such as toluene, hexane, acetone, and diethyl ether. The capillary condensation causes the reflectivity shifted to longer wavelengths which is characteristic of an increase in the average refractive index of the multilayers. This consistent with the replacement of a significant amount of empty pore volume with analyte. The smart particles terminated with silicon hydride functionalities were displayed the most red-shifted photonic feature for the analyte having the highest vapor 
pressure, indicating that the specificity of adsorption or capillary condensation at the PSi surfaces depends dramatically on the vapor pressure.

\section{References}

[1] M. D. Kelzenberg, D. B. Turner-Evans, B. M. Kayes, M. A. Filler, M. C. Putnam, N. S. Lewis, and H. A. Atwater, "Photovoltaic measurements in single-nanowire silicon solar cells", Nano Lett. Vol. 8, pp. 710-714, 2008.

[2] Y. Cui, Z. H. Zhong, D. L. Wang, W. U. Wang, and C. M. Lieber, "High performance silicon nanowire field effect transistors", Nano Lett. Vol. 3, pp. 149152, 2003.

[3] D. H. Jung, "Biosensor based on distributed bragg reflector photonic crystals for the detection of protein A", J. Chosun Natural Sci., Vol. 3, pp. 33-37, 2010.

[4] S. H. Jang, "Chemical and Physical Properties of Porous Silicon”, J. Chosun Natural Sci., Vol. 4, pp. 1-6, 2011.

[5] S. G. Kim, "Optical characterization of smart dust based on photonic crystals and its sensing applications", J. Chosun Natural Sci, Vol. 4, pp. 7-10, 2011.

[6] Y. D. Koh, "1-D Photonic crystals based on bragg structure for sensing and drug delivery applications", J. Chosun Natural Sci., Vol. 4, pp. 11-14, 2011.

[7] K. S. Jung, "Fabrication and characterization of DBR porous silicon chip for the detection of chemical nerve agents", J. Chosun Natural Sci, Vol. 3, pp. 237-240, 2010.

[8] S. D. Cho, "Detection of nitroaromatic compounds with functionalized porous silicon using quenching photoluminescence", J. Chosun Natural Sci., Vol. 3, pp. 202-205, 2010.

[9] S. H. Jang, "Study on thickness of porous silicon layer according to the various anodization times", J. Chosun Natural Sci., Vol. 3, pp. 206-209, 2010.

[10] S. H. Jang, "Investigation of relationship between etch current and morphology and porosity of porous silicon", J. Chosun Natural Sci., Vol. 3, pp. 210-214, 2010.

[11] J. M. Han, "Photoluminescence of porous silicon according to various etching times and various applied current densities", J. Chosun Natural Sci., Vol. 3, pp. 148-152, 2010.

[12] Y. D. Koh, "Analysis on oxidation of porous silica obtained from thermal oxidation of porous silicon", J. Chosun Natural Sci, Vol. 3, pp. 153-156, 2010.

[13] V. S.-Y. Lin, K. Motesharei, K.-P. S. Dancil, M. J. Sailor, and M. R. Ghadiri, "A porous silicon-based optical interferometric biosensor", Science, Vol. 278, pp. 840-843, 1997.

[14] A. Janshoff, K.-P. S. Dancil, C. Steinem, D. P. Greiner, V. S. Y. Lin, C. Gurtner, K. Motesharei, M. J. Sailor, M. R. Ghadiri, "Macroporous p-type silicon Fabry-Perot layers. Fabrication, characterization, and applications in biosensing", J. Am. Chem. Soc., vol. 120 no. 46, pp. 12108-12116, 1998.

[15] D. van Noort, S. Welin-Klinstrom, H. Arwin, S. Zangooie, I. Lundstrom, and C.-F. Mandnius, "Monitoring specific interaction of low molecular weight biomolecules on oxidized porous silicon using ellipsometry", Biosens. Bioelectro., Vol. 13, pp. 439-449, 1998.

[16] S. Chan, S. R. Horner, B. L. Miller, and P. M. Fauchet, "Identification of Gram negative bacteria using nanoscale silicon microcavities", J. Am. Chem. Soc., Vol. 123, pp. 11797-11798, 2001.

[17] B. E. Collins, K.-P. S. Dancil, G. Abbi, and M. J. Sailor, "Determining protein size using an electrochemically machined pore gradient in silicon", Adv. Funct. Matter., Vol. 12, pp. 187-191, 2002. 